\title{
Zur internationalen Entwicklung in der Geographiedidaktik
}

\section{Vorbemerkung:}

Über die internationale Entwicklung in der Geographiedidaktik berichten zu können, ist recht schwierig. Sprachbarrieren und unvollständige globale Kommunikation erschweren den Überblick. Außerdem sind zahlreiche regionale Situationen, wie z. B. in der Arabischen Welt oder auch in Lateinamerika, entweder nicht zugänglich oder schier unüberschaubar.

Daß der Autor dieses Berichts auf die entsprechende Anfrage der Herausgeberin dieses Heftes trotzdem zugesagt hat, beruht auf folgenden Ursachen:

- Er arbeitet seit einigen Jahren auf dem Gebiet der international vergleichenden Geographiedidaktik.

S. HAUBRICH, H. (Hrsg) (1982): Geographische Erziehung im internationalen Blickfeld; erhältlich durch: Georg-Eckert-Institut, Cellerstr. 3, D-3300 Braunschweig, und Haubrich, H. (Hrsg) (1987): International Trends in Geographical Education; erhältlich durch H. Haubrich, Birkenrain 34, D-7811 St. Peter/Germany FR

- Er nahm an den jüngsten Symposia der Internationalen Geographischen Union in Freiburg/Paris 1984, Sitges/Barcelona 1986, und Brisbane/Sydney 1988 teil und konnte z. B. in Sydney die Berichte über die Entwicklung der Geographiedidaktik bzw. des Geographieunterrichts aus über 30 Ländern anhören und mit den betreffenden Berichterstattern sprechen.

- Er wurde während des Kongresses der Internationalen Geographischen Union in Sydney 1988 von der Generalversammlung zum Vorsitzenden der Kommission «Geographical Education» gewählt und arbeitet nun mit zahlreichen Kommissionsmitgliedern aus vielen Ländern zusammen, um die geographiedidaktische Forschung voranzutreiben und den Geographieunterricht zum Wohle der heranwachsenden Generation zu stärken.

Aus der Fülle der vorliegenden Informationen, aus denen sich kaum gemeinsame globale Trends ableiten lassen, sollen nun folgende nationale oder regionale Entwicklungen ausgewählt werden:
1. «Perestroika» im Geographieunterricht der UdSSR

2. «Renaissance» des Geographieunterrichts in den USA

3. Im Labyrinth des Geographieunterrichts in Europa

4. Endogene Philosophie des Geographieunterrichts in einem Entwicklungsland

5. Struktur und Projekte der Kommission «Geographical Education» der Internationalen Geographischen Union für die Periode 1988 bis 1992.

\section{1. «Perestroika» im Geographieunterricht der UdSSR}

Grundlage dieses Abschnittes ist ein Bericht von Professor Vladimir Maksakovsky, Mitglied der Akademie der Wissenschaften und Lehrer an der Pädagogischen Hochschule in Moskau, über den jüngsten Wandel im Geographieunterricht der Sowjetunion. Dieser Bericht wurde im August 1988 in Brisbane/Australien vorgetragen und soll nun im folgenden gekürzt sinngemäß wiedergegeben werden:

Das russische Wort «Perestroika» ist heutzutage in aller Munde. Es charakterisiert den Prozeß der Reform, die sich zur Zeit in der sowjetischen Gesellschaft ereignet. Perestroika hat alle Sphären des Lebens - die Schule eingeschlossen - ergriffen.

In der Sowjetunion gibt es 138000 Schulen, 55 Millionen Schüler und über drei Millionen Lehrer.

1984 begann man mit einer Schulreform, die den Sinn hatte, die Einheitsschulen der wissenschaftlichen, technologischen und sozialen Entwicklung der Gegenwart anzupassen. Lehrpläne wurden revidiert, neue Schülerbücher und Lehrerhandbücher geschrieben. Die meisten Schüler gingen nun vom 6. Lebensjahr an nicht zehn, sondern sogar elf Jahre in die Schule. Während dieses Prozesses tauchte die Perestroika-Diskussion auf, die nun Politik, Wirtschaft, Management, Ideologie und Psychologie der Gesellschaft erfaßte. Gorbatschow ging die

Hartwig Haubrich, Prof., Dr., Pädagogische Hochschule Freiburg i. Br., Kunzenweg 21, D-7800 Freiburg 
Schulreform nicht weit genug, und er machte im Februar 1988 die sowjetische Schule zum Gegenstand der Tagesordnung einer Plenarsitzung des Zentralkomitees der KPdSU. Das Ergebnis waren politische Leitlinien bzw. das gegenwärtige Grundkonzept für alle Sekundarschulen in der Sowjetunion.

Demokratisierung des Schullebens wurde nun zur Leitidee sowohl für die Schulleitung als auch für das Verhältnis zwischen Lehrern und Schülern. Eine «kooperative Pädagogik» sollte die zukünftige Schule auszeichnen. Außerdem wurde ein «lebenslanges Lernen» gefordert, für das in der Schule durch ein «Lernen lernen» Grund gelegt werden sollte. Das Konzept der «Einheitsschule», die allen das Gleiche bietet, wurde grundsätzlich abgelehnt. Die zukünftige Schule soll den individuellen Fähigkeiten und Interessen der Schüler mehr als bisher gerecht werden. Aus diesem Grunde sind Schulen verschiedenen Typs bzw. verschiedener Schwerpunkte für verschiedenartige Schülergruppen gefordert. Insbesondere sollen Schüler der 10. und 11. Klasse zahlreiche Wahlmöglichkeiten entsprechend ihrer individuellen Interessen erhalten. Im Gegenzug werden die verpflichtenden Lehranteile des Curriculums reduziert.

Ein weiteres Konzept der neuen Schule wird als «Humanisierung der Bildung» bezeichnet, die den Menschen auch als Individuum in den Mittelpunkt stellt. Dies bringt auch eine Stärkung der Lehrerpersönlichkeit mit, die als Zentralfigur im Perestroika-Prozeß der Schule angesehen wird. Vermehrt werden curriculare Entscheidungen in die Hand regionaler Lehrergruppen und Verwaltungsentscheidungen in die Kompetenz von Lehrerkonferenzen gegeben. Der Perestroika-Prozeß der Schule wurde nicht nur von oben gefordert, sondern erfuhr eine breite Diskussion in der Öffentlichkeit, was als natürlich bezeichnet wird, da die meisten Familien durch ihre Kinder eng mit der Schule verbunden sind.

Auch die Schulgeographie blieb nicht von der Perestroika-Diskussion verschont. Obwohl die sowjetische Schulgeographie auf alte Traditionen zurückgeht, wurde sie doch sehr stark kritisiert. Vor allem die Lehrerschaft beklagte die Stoffülle der Curricula, die allerdings vom Berichterstatter als nur natürlich bezeichnet wurde, da der Geographieunterricht schließlich über die ganze Erde unterrichten müsse. Die Fachwissenschaftler kritisierten den Rückstand der Schulgeographie im Vergleich zu den geographischen Forschungsergebnissen und vor allem die mangelnde Verbindung zwischen Natur- und Wirtschaftsgeographie. Diese gravierenden Defizite erforderten deshalb eine grundsätzliche Erneuerung der sowjetischen Schulgeographie. Die neuen Ziele und Inhalte auch des Geographieunterrichts sollen eine allumfassende, harmonisch entwickelte Persönlichkeit des Schülers anstreben.
Nicht die Ausbildung von Geographen, sondern die Erziehung für die Notwendigkeit des Lebens vorbereiteter Menschen ist gefragt. Seine Allgemeinbildung besteht aus Grundkenntnissen der Mathematik, der Chemie, der Biologie, der Geographie, der Geschichte usw., so daß er sich ein universelles, sachgerechtes Bild von der Welt machen kann. Die geographische Bildung wird als ein wichtiger Bestandteil einer allumfassenden Kultur betrachtet, die eine wissenschaftliche Erklärung für eine große Zahl von Phänomenen und Prozessen in der Natur und Gesellschaft anbieten kann. Deshali gehören geographische und karthographische Kenntnisse, aber auch Fähigkeiten wie z.B. geographisches Denken und die Anwendung geographischer Begriffe zum Kerncurriculum der allgemeinbildenden Schulen.

Ein wichtiges Problem der Geographiedidaktik war es, zwischen einer allgemeinen und einer speziellen geographischen Bildung zu unterscheiden und entsprechende Inhalte auszuwählen, die für alle $45 \mathrm{Mil}$ lionen sowjetischer Schüler von grundlegender Bedeutung zur Bewältigung ihrer Zukunft sind und diejenigen geographischen Inhalte, die nur für speziell Interessierte angeboten werden sollten. Zu den grundlegenden Bildungsinhalten zählen z.B. Kartenkunde, Geozonen, natürliche Ressourcen, Verstädterung, Weltwirtschaft, geographische Arbeitsteilung usw. Während früher der Schüler bei der Behandlung Australiens sieben Klimatypen zu unterscheiden lernte, wird dies im neuen Lehrplan als irrelevant betrachtet und deshalb nicht berücksichtigt. Die neuen inhaltlichen Akzente liegen bei aktuellen Themen und Tendenzen wie z. B. ökologischen, gesellschaftlichen und wirtschaftlichen Entwicklungen.

Außerdem verfolgt die Schulgeographie nun folgende mehr instrumentale Ziele:

- komplexe, synthetische bzw. integrative Betrachtungsweisen zu stärken,

- nomothetische, d.h. allgemeingeographische Vorgehensweisen auf Kosten idiographischer, d. h. regionalgeographischer Studien vorzuziehen.

Der jüngste Lehrplan hat nun folgende Sequenz erhalten:

6. Klasse: Basiskurs in Physischer Geographie (68 Perioden),

7. Klasse: Geographie der Kontinente und Ozeane (102 Perioden),

8. Klasse: Geographie der UdSSR (68 Perioden),

9. Klasse: Geographie der UdSSR (68 Perioden),

10. Klasse: Wirtschafts- und Sozialgeographie der Welt (51 Perioden).

Vor allem die zehnte Klasse wurde völlig neu gestaltet. Im Gegensatz zum vorhergehenden Lehrplan, der einen Kurs zur Wirtschaftsgeographie fremder 
Länder vorsah, sind die neuen Inhalte kaum noch auf einzelne Länder bezogen (da diese Gegenstand der 7. Klasse darstellen), sondern allgemeine wirtschafts- und sozialgeographische Strukturen und Prozesse der Welt, natürliche Ressourcen, ökologische Probleme, Weltbevölkerung, Weltwirtschaft, Schicksal der Menschheit sind die Kerninhalte der 10. Klasse geworden.

Auch die methodischen Vorgehensweisen im Geographieunterricht haben sich geändert. Hauptanliegen ist die Konzentration auf substantiell bedeutsame Inhalte. Sekundäres wird aufgegeben. Die Erarbeitung von Zusammenhängen bzw. die Berücksichtigung von Theorien und Modellen erhält den Vorzug vor der Last der Fakten. Problemorientierung, Schülerorientierung, Individualisierung, Gebrauch technischer Medien, aber auch Wissenschaftsorientierung sind die Maximen einer neuen Methodik. Dabei werden drei Formen des Lernens neuer Inhalte unterschieden:

1. Lernen von Inhalten und Fähigkeiten in didaktisch zubereiteter Form,

2. Anwenden von Erlerntem in ähnlichen Situationen,

3. Individuelle kreative Tätigkeit in völlig unbekannten Bereichen.

Vor allem die zweite und dritte Form werden als pädagogisch besonders wertvoll betrachtet .

Inhaltlich soll dabei dem Typischen bzw. Exemplarischen sowohl bei Erscheinungen als auch in Prozessen der Vorzug gegeben werden. Das exemplarische Prinzip führt sogar soweit, daß in Zukunft nicht alle 16 Wirtschaftsregionen der UdSSR als zentraler Gegenstand des Geographieunterrichts studiert werden müssen, sondern man beschränkt sich auf drei Hauptwirtschaftszonen im Westen, Osten und Südosten der Sowjetunion. Nicht Details, sondern Exemplarisches steht im Mittelpunkt. Wissenschaftsorientierung und Schülerorientierung, d. h. Exaktheit der inhaltlichen Aussage und die Fähigkeit des Schülers, diese zu begreifen, sollen in Übereinstimmung gebracht werden. Die Beziehung zwischen Inhalt und Form pädagogisch zu bewältigen, ist die Herausforderung an den Geographiedidaktiker, der die Medien sowohl motivierend als auch verständlich gestalten soll. Lernen soll interessant werden. Der Berichterstatter wies auch darauf hin, daß westliche Erfahrungen Eingang in den Prozeß der Perestroika in der Schulgeographie gefunden hätten. Dabei dienten vor allem folgende Kanäle: 1. die Kommission Geographical Education der Internationalen Geographischen Union und 2. die bilateralen Schulbuchkommissionen mit deṇ USA, der Bundesrepublik, Österreich und anderen sozialistischen und kapitalistischen Ländern.

Dabei seien unter anderem Konzepte wie der fragend entwickelnde Unterricht, entdeckendes Lernen, Wahl und Präsentation von Fallstudien, Plura- lismus von Schülerbüchern, Arbeitsbüchern, interdisziplinäre Kurse, Anwendung von Computern und audiovisuelle Medien von besonderem Interesse. Insbesondere sei hierbei die russische Übersetzung des Buches «New UNESCO Source Book for Geography Teaching» bedeutsam gewesen. Im Gegensatz dazu wird bedauert, daß die westliche Seite weder von der fachwissenschaftlichen noch von der fachdidaktischen Literatur der Sowjetunion Notiz nimmt. Selbst die geographischen Schülerbuchkapitel über die Sowjetunion wären voller statistischer und anderer Fehler, veraltet und nicht geeignet, die räumliche Entwicklung in der Sowjetunion angemessen darzustellen. Noch bedauerlicher jedoch seien die dargestellten Vorurteile und Stereotypen, die die Sowjetunion als ein kaltes, isoliertes, rückständiges Land darstellten, wenn nicht sogar als «Reich des Bösen» betrachteten. Um die Vorurteile sowohl in sowjetischen als auch in westlichen Schülerbüchern zu eliminieren und ein gegenseitiges Verstehen der Nationen zu ermöglichen, wird ein neues politisches, aber auch pädagogisches Denken im Sinne einer Perestroika als notwendig erachtet.

\section{2. «Renaissance» des Geographieunterrichts in den USA}

Grundlage dieser Darlegung sind die Berichte von sieben Geographiedidaktikern aus der sogenannten «Alliance Movement» in den USA während des Precongress Symposiums in Brisbane/Australien im August 1988 und die "Guidelines for Geographic Education», Washington 1986.

Wohl wegen der Integration des Faches Erdkunde in den Fachbereich «Social Studies» (zusammen mit Geschichte und Politik) und trotz des in der internationalen Geographiedidaktik hoch angesehenen «High School Geography Project» (HSGP) hat die Schulgeographie in den USA einen unvergleichlichen Niedergang erfahren. Ihr Anteil am Gesamtcurriculum näherte sich - wenn auch von Staat zu Staat verschieden - häufig dem Wert Null. Selbst heute mangelt es immer noch am Stundendeputat und nicht zuletzt an ausgebildeten Geographielehrern. Zahlreiche Umfragen der letzten Jahre belegten eine für eine Weltmacht beschämende geographische Unkenntnis. * Selbst in den entsprechenden Krisenzeiten konnte der sogenannte «Mann auf der Straße» weder Korea, noch Vietnam, noch Gre-

\footnotetext{
1983 konnten nur 20\% von zwölfjährigen Schülern die USA auf einer Weltkarte lokalisieren, über 20\% bezeichneten Brasilien als die Vereinigten Staaten.
} 
nada oder andere Krisenherde geographisch einordnen. Immer wieder vorgenommene Umfragen der Bevölkerung durch das Gallup-Institut (1981-1985) über als notwendig erachtete schulische Inhalte ergaben wenig geographisch Fundamentales, sondern nach den bekannt gewordenen und in der Presse diskutierten erschreckenden Tests vorwiegend topographische Kenntnisse oder Wissen über Länder und Völker.

In dieser Notsituation haben sich Geographen von Hochschule und Schule, von Verlagen, Planungsbehörden und anderen angewandten Feldern zur schon genannten «Alliance Movement» zusammengetan, um der Geographie in der Schule eine Auferstehung aus der Asche zu ermöglichen. So entstanden in zahlreichen Staaten Ausbildungszentren für Multiplikatoren - meist "summer schools»-, die wiederum anderen Lehrern in ihren Schulen oder Schulregionen mit Rat und Tat zur Seite stehen sollen. Aber selbst die Multiplikatoren sind oft nicht als Geographen an Universitäten ausgebildet worden. Die Aufgabe der Alliance Movement ist riesig, der Enthusiasmus der Geographen beeindruckend, ebenso wie die finanzielle Unterstützung durch die Privatwirtschaft, insbesondere durch die Zeitschrift National Geographic, die viele Millionen Dollar für die Renaissance des Geographieunterrichts, d. h. für die Multiplikatoren-Zentren zur Verfügung gestellt hat.

Grundlage der Arbeit sind die «Guidelines for Geographic Education» 1986, die gemeinsam von den Verbänden der Universitäts- und Schulgeographen (National Council for Geographic Education and Association of American Geographers) auf nationaler Ebene entwickelt wurden, obwohl die einzelnen Staaten eine eigene Bildungsautonomie besitzen Wenn es auch interessant wäre, die Suche nach fachpolitisch wirksamen Strategien zu beschreiben, so interessiert in diesem Zusammenhang wohl mehr das curriculare Konzept, das in den Multiplikatorenzentren Gegenstand der Kurse darstellt und ebenso als Grundlage zur Entwicklung neuer Medien benutzt wird.

Trotz der vorhandenen Unkenntnisse gehen die Guidelines nicht von einer vorwiegenden Kenntnisvermittlung aus - Kenntnisse werden zwar als notwendig erachtet -, sondern von einem wissenschaftlichen Vorgehen, Kenntnisse zu erwerben (d. h. nicht zu erfahren, wo etwas ist, sondern auch, warum es dort ist und wo es eigentlich sein sollte). Die erste Aufgabe der Geographie sei es, Orte zu lokalisieren, zu beschreiben und ihre natürlichen und kulturellen Erscheinungen zu erklären; zu erforschen, wie Menschen auf ihre Umwelt reagieren und sie formen. Suchinstrumenten zum Sammeln räumlicher Daten, zur Darstellung und zur Interpretation dieser Daten werden höchste Priorität eingeräumt.
Interessant und anregend ist die sogenannte «concept»-Orientierung des Lehrplans. Alle Unterrichtsthemen sollen sich wenigstens an einem der folgenden Konzepte orientieren:

1. Lage

2. Ort (place)

3. Mensch-Raum-Beziehungen

4. Interaktion

5. Region

$Z u$ 1: Unter Lage wird die absolute und relative Lage verstanden. Die absolute Lage ist die unveränderliche Lage auf dem Längen- und Breitenkreis des Gradnetzes. Die relative Lage ist dagegen veränderlich und im lokalen, regionalen, nationalen und globalen Maßstab jeweils abhängig von Veränderungen und Prozessen wie z. B. dem globalen Versorgungsstrom an Energie wie Öl und Gas oder an bestimmten Nahrungsmitteln. Die kleinräumliche topographische Lage und die großräumliche geographische Lage machen die Lage - und damit Lebensbedingungen eines Ortes aus.

$Z u$ 2: Die Orte der Erde sind durch verschiedene, d. h. physische und kulturelle Eigenschaften ausgezeichnet. $\mathrm{Zu}$ den physischen Merkmalen zählen geologische, hydrologische, klimatische und biologische; kulturelle Eigenschaften werden durch menschliche Ideen und Handlungen geschaffen. Bevölkerungsstruktur, Siedlungsmuster, Wirtschaftsformen, Freizeit, Verkehr und Kommunikation kennzeichnen die kulturelle Beschaffenheit eines Ortes. Sie basieren auf der Ideologie, Philosophie, Religion, Sprache und gesellschaftlichen Organisationen der Menschen. Die Eigenschaften eines Ortes werden von der Bevölkerung in der Regel nur selektiv wahrgenommen. So wird z. B. Zentralamerika hier als ein tropisches Bergland betrachtet, dort als ein Gebiet sozialer und politischer Unruhen oder als ein Vulkangebiet, ein Land tropischer Kulturpflanzen, eine spanisch-indianische Kulturregion, eine Ansammlung kleiner unabhängiger Staaten bzw. als eine Ansammlung typischer Entwicklungsländer. Die Wahrnehmung der Eigenschaften eines Raumes bleibt in der Regel selektiv und perspektivisch.

Zu 3: Alle Orte der Erde bieten Vorzüge und Nachteile für eine menschliche Besiedlung. Flußniederungen mit fruchtbaren Böden und Wüsten mit Trockenheitenklimaten stellen jeweils eine andere Herausforderung an die Menschen dar. Trotzdem ist die Reaktion menschlicher Gruppen nicht determiniert, wie es z.B. menschenleere Wüsten hier und die Kultivierung der Wüste Negev in Israel dort belegen. Je nach wirtschaftlichen, politischen und technischen Möglichkeiten bzw. Werthaltungen der Gesellschaft verändern die Menschen ihr Naturmilieu in verschiedener Weise. So können gleiche na- 
türliche Rahmenbedingungen von verschiedenen Kulturen verschiedenartig in Wert gesetzt werden.

Zu 4: Die Menschen leben hier in Städten, dort in Dörfern, hier in dicht und dort in dünn besiedelten Gebieten. Aber sie interagieren miteinander durch Reisen und Begegnungen bzw. durch den Austausch von Waren und Ideen. Der augenscheinlichste Beleg für die globale Abhängigkeit der Menschheit sind die großen internationalen Verkehrslinien und Ströme. Sie beweisen, wie die Völker und Nationen täglich kooperieren und nicht mehr autark sind. Räumliche Interaktionen beziehen sich auch auf den engen Lebensraum wie z. B. der tägliche Schulweg, die Einkaufsfahrt zum nächsten Markt, das Pendeln zum Arbeitsplatz oder zum Naherholungsraum. Neue technologische und politische Bedingungen, aber auch Veränderungen menschlicher Werthaltungen führen zu neuen räumlichen Interaktionsmustern.

Zu 5: Die Grundeinheit einer jeden geographischen Studie ist die Region, die durch ein einheitliches Kriterium definiert wird. Nation, Provinz, Land oder Stadt sind solche augenfälligen Regionen, andere werden durch eine einheitliche Sprache, eine einheitliche Verwaltung, eine einheitliche Landschaft geformt oder durch ein Zusammenspiel mehrerer Eigenschaften gebildet. Auch Stadtviertel, hier mit Schichten hoher Einkommen, dort mit Schichten niedriger Einkommen bzw. Hauptgeschäftszentren zählen zu solchen räumlichen Einheiten. Didaktisch bilden Regionen überschaubare Kenntniseinheiten, sie bieten den Kontext zu Studien aktueller Prozesse und bilden ein Bindeglied zwischen den partiell-regionalen und ganzheitlichglobalen Weltbildern des Schülers. Regionen sind ein Hilfsmittel, um die Erde als ein integratives planetarisches Ökosystem zu begreifen.

Es würde zu weit führen, um an dieser Stelle über den Lehrplan mit seinen zahlreichen Lernerwartungen zu referieren. Um aber doch einen Eindruck von der Struktur des neuen Geographiecurriculums zu geben, werden im folgenden die Leitziele (central focus), regionalen Schwerpunkte und vor allem die jeweils zu beachtenden Konzepte aufgeführt.

Kindergarten bis zur 2. Klasse: Ich selbst im Raum; Lage, Ortseigenschaften

Wohnung/Wohnhaus und Schule an verschiedenen Orten, relative Lage

Eigenschaften der näheren Umwelt

Nachbarschaften - kleinere Gebiete in größeren Gemeinden

Lage, Ort, Umweltveränderungen, Abhängigkeiten im Raum, Interaktionen innerhalb und zwischen Nachbarschaften, Nachbarschaften als Gebiete mit Ähnlichkeiten und Unterschieden
Klasse 3 und 4:

Gemeinde - Raum mit anderen Teilen:

relative Lage, Ausstattung der Landschaft, Umweltbeziehungen, Abhängigkeiten und Interaktionen in einer Gemeinde, die Gemeinde als eine Region

Der Staat, die Nation und die Welt

Lage, die Natur und die Eigenschaften von Orten

Interaktionen zwischen Kultur- und Naturlandschaft, menschliche Interaktionen innerhalb und zwischen Staat, Nation und Welt, globale Abhängigkeit, die Natur einer Region

\section{Klasse 5 und 6:}

Vereinigte Staaten, Canada und Mexiko:

Lage, vergleichende Analyse von Gebieten, Lebensqualität, menschliche Interaktionen in den USA mit Canada und Mexiko, Natur- und Kulturlandschaften in den USA, in Canada und Mexiko, Lateinamerika, Europa, UdSSR, der Mittlere Osten, Asien und Afrika.

Lage, natur- und kulturgeographische Ausstattung, Mensch-Umwelt-Beziehungen, räumliche Interaktionen, Regionen und Subregionen der Erde

Klasse 7-9:

Staat oder Regionalgeographie:

Mensch-Raum-Beziehungen, Lebensqualität, regionale Strukturen

Weltgeographie:

globale Muster physisch- und kulturgeographischer Erscheinungen, Beziehungen zwischen Regionen, Konflikte

Klasse 8-10:

Geowissenschaften: natürliche Umwelt, ErdeSonne-Beziehungen, Energiefluß, hydrologischer Kreislauf, tektonische und erosive Prozesse, Wetter, Landformen, Klimatypen, Ozeanographie Vereinigte Staaten von Amerika

Systematische Regionalanalyse: Entwicklung des Staates als Nation, Raumeigenschaften, menschliche Interaktionen, räumliche Beziehungen, vergleichende Analyse von Subregionen

Klasse 9-12:

Umweltgeographie: Umweltmanagement im lokalen, nationalen, globalen Maßstab, Ökosysteme, Umweltwahrnehmungen, Umweltqualität, Umweltschutz, Natur- und Sozialkatastrophen

Stadtgeographie: zentrale Orte, metropolitane Gebiete, Interaktionen von Menschen, Transport von Gütern und Ideen, geschichtliche und kulturelle Strukturen

\section{Politische Geographie:}

Konflikte, Wahlgeographie, Raumplanung, internationale Zusammenarbeit

Kulturgeographie:

Kulturen, Kulturlandschaft als Individualität, Siedlungsformen, lokale und regionale kulturgeographische Eigenheiten 
Historische Geographie:

historische Landschaft im kolonialen Amerika im Mittelosten, im Islam, in Lateinamerika, Interaktionen mit der Dritten Welt

Wirtschaftsgeographie: Ausnützung der natürlichen Ressourcen, Handel und Verkehr, Dienstleistungen, Lebensqualität

Probleme im lokalen Umfeld der eigenen Gemeinde

Erfassen lokaler Probleme, Sammeln und Analysieren von Daten, Entwickeln eigener Lösungsmodelle und Einsatz im politischen Rahmen

Leistungskurse in den Klassen 11 und 12:

Forschungsorientierte Projekte eigener Wahl aus der Kultur- oder Physischen Geographie, z. B. Bevölkerungsstruktur, Kommunikation, Ortsanalyse, Karten- und Luftbildinterpretation, Umweltanalysen (vorwiegend selbständige Forschungsarbeit der Schüler unter Anleitung eines Lehrers)

Nach der Auflistung der Ziele und Inhalte für die einzelnen Schulstufen, die hier nur teilweise wiedergegeben werden konnten, folgt eine weitere Zusammenstellung von Fähigkeiten, die besondere Berücksichtigung im praktischen Unterricht erfahren sollen. Diese Fähigkeiten werden in die folgenden fünf Gruppen gegliedert:

A. Geographische Fragen stellen

B. Geographische Informationen finden

C. Geographische Informationen darstellen

D. Geographische Informationen analysieren

E. Geographische Generalisierungen induktiv und deduktiv formulieren und testen.

Diese von den beiden großen amerikanischen Geographiegesellschaften gemeinsam erarbeiteten Rahmenrichtlinien dienen zur Zeit als Grundlage für Multiplikatorenkurse, in denen sowohl das Gesamtkonzept geklärt wird, aber gleichzeitig auch konkrete Unterrichtseinheiten und -materialien entwikkelt werden. Dieser in Amerika erst- und einmalige Vorgang läßt hoffen, daß es der Alliance Movement gelingt, die Renaissance des Geographieunterrichts und damit die geographische Bildung der Bevölkerung mit Hilfe der Zeitschrift «National Geographic» auf den Weg zu bringen.

\section{Das Labyrinth «Geographieunterricht» in Europa}

Grundlage dieser Darstellung sind eigene Recherchen und Gespräche vor Ort und nicht zuletzt das sogenannte «Mitteleuropäische Geographiedidaktik Symposium» während des Deutschen Schulgeographentages in Salzburg 1988.
Sucht man nach dem Fach Geographie in Europa, so findet man es nicht sehr oft als selbständiges Fach, d. h. als Erdkunde oder Geographie. Häufig steht es im Verbund mit anderen Fächern, wie z. B. in Österreich als Geographie und Wirtschaftskunde, in Spanien als Geographie und Geschichte, in Frankreich als Geographie, Geschichte und Staatsbürgerkunde, in Niedersachsen als Weltkunde gemeinsam mit Geschichte und Gemeinschaftskunde, in Großbritannien als social studies gemeinsam mit Geschichte, Gemeinschaftskunde und religiöser Erziehung. Vergleicht man nun die verfügbaren Stunden für das Fach Erdkunde, so findet man auf gleichen Klassenstufen 0 bis 3 Wochenstunden, und auch das gesamte Stundendeputat für die Sekundarstufe I und II differiert zwischen 8 und 16 Wochenstunden.

Der Vergleich der Inhalte auf den verschiedenen Stufen ist nicht mehr überschaubar und darstellbar. Auch die Ziele setzen völlig verschiedene Akzente. So beziehen sich z. B. die Hauptziele des Geographieunterrichts in der Lehrplanpräambel Italiens auf die «Humanistische und christliche Tradition, die die Menschenwürde und die Achtung vor Werten der Geistigkeit und Freiheit für eine grundlegende Bildung anerkennt». Polen betrachtet den Geographieunterricht in seiner Funktion durch eine «rationale und kritische Einschätzung der Wechselbeziehungen zwischen Natur und Gesellschaft bzw. des Mensch-Umwelt-Systems». Als eine «ausgezeichnete Arznei gegen Voluntarismus und nationale Megalomanie einerseits und gegen Minderwertigkeitskomplexe andererseits», weiter gegen "Chauvinismus und Rassenvorurteile bzw. zur Schaffung der psychologischen Voraussetzungen für die Achtung und Verständigung zwischen Nationen und Gesellschaft». Während z. B. englische Lehrer ihre geographischen Lernziele sehr unterschiedlich definieren, sehen Geographen sozialistischer Länder ihre Aufgabe im Rahmen der «sozialistischen Menschenbildung» klar und prägnant. Ein britischer Kollege meint: «Der durchschnittliche Lehrer ist geneigt, pragmatisch zu lehren.» Währenddessen wird in der Tschechoslowakei die politisch-ideologische Funktion der geographischen Erziehung hervorgehoben, die aufgrund ihrer speziellen Fragestellung das «sozialistische Menschenbild» zu gestalten bzw. einen Beitrag zu leisten habe - «zum materialistischen Weltbild». Durch die Beachtung des «dialektischen Verhältnisses von Natur und Gesellschaft», des «Kampfes gegen egoistische Geopolitik und Rassismus» und der «marxistischen Philosophie und politischen Ökonomie», um das «Verhältnis zu der sozialistischen Staatengemeinschaft, die Liebe zur Heimat, sozialistischen Patriotismus und Internationalismus» fortzuentwickeln. In der DDR soll der Geographieunterricht «Verständnis für den Prozeß der gesellschaftlichen Entwicklung wecken und zu der Erkenntnis führen, daß nur die 
sozialistische und kommunistische Gesellschaft unter der Führung der Arbeiterklasse und ihrer Partei die freie Entwicklung der Schöpferkraft des Menschen garantiere, Wissenschaft und Technik und die Reichtümer der Natur zum Wohle des Menschen ausnutze». Damit wird die überwiegend politische und ideologische Auslegung des Geographieunterrichts deutlich. Er soll zusammen mit den anderen Fächern bei der Gestaltung der entwickelten sozialistischen Gesellschaft im real existierenden Sozialismus mitwirken» (siehe SPERLING 1977 und BARTH in: HAUBRICH 1987).

Lehrplanreformen sind zu jeder Zeit in mindestens einigen europäischen Ländern zu beobachten. Der Reform in der Sowjetunion, die vorher skizziert wurde und offenkundig Dezentralisierungsbestrebungen erkennen läßt, steht eine Zentralisierung in Großbritannien gegenüber. Das klassische Land der «school-based curricula», Großbritannien, wo die Lehrer seit vielen Jahrzehnten auf lokaler oder regionaler Basis gewohnt waren, ihre orts- und situationsgerechten Lehrpläne zu machen, steht vor der Aufforderung der Regierung, nationale Lehrpläne zu schaffen, die für alle Schulen verpflichtend gelten. Neben der Gefahr der Egalisierung der Ziele und Inhalte sehen die britischen Geographen noch eine größere Drohung, nämlich das Ausscheiden des Geographieunterrichts aus dem Kerncurriculum. Die Dezentralisierung in der Sowjetunion und die Zentralisierung in Großbritannien sind wahrhaft Entwicklungen, die nicht den üblichen Wahrnehmungen und klassischen Bildern dieser beiden Länder entsprechen. Wie offen und flexibel trotzdem Lehrpläne sein können, belegt die Beobachtung eines Pädagogen, der behauptet, in den Schulen Frankreichs würden viele Lehrer anders unterrichten als es vom zentralistischen Lehrplan verlangt wird, während im föderalistischen System Großbritanniens viele Lehrer trotz der dezentralen Lehrpläne entsprechend dem aktuellen Zeitgeist das Gleiche unterrichten. Die Relativierung der Wirksamkeit der Lehrpläne weist auf die Bedeutung der Lehrerpersönlichkeit hin. Diese bedarf allerdings der Hilfe. Überall dort, wo Hochschullehrer und Lehrer zusammenarbeiten - wie es Klaus Aerni für die Schweiz während des Mitteleuropäischen Geographiedidaktik Symposiums in Salzburg 1988 berichtet - ist es um den Geographieunterricht grundsätzlich besser bestellt. Auffälligerweise scheint dieser Tatbestand in sozialistischen Ländern Europas eher gegeben als in westlichen Ländern.

Abrupte Reformen haben häufig mehr negative als positive Wirkungen, und deshalb plädiert z. B. Ludwig Barth aus der Deutschen Demokratischen Republik für eine kontinuierliche Reform des Geographieunterrichts von innen heraus und nicht für plötzliche Innovationsschübe. Die Legitimation geographischer Inhalte ist in westlichen Ländern in der Regel ein größeres Problem als in den östlichen. Wenn z. B. der niederländische Kollege Wim Veen im Rahmen des o. a. Symposiums verlangt, endlich dem Schüler das anzubieten, was er wirklich im Leben braucht, und damit vor der Schwierigkeit steht, diese Inhalte zu bestimmen, weist Günter Kirchberg aus der Bundesrepublik Deutschland nach Klafki auf die die Persönlichkeit erschließenden Inhalte hin und steht damit vor der schier unlösbaren Schwierigkeit, pädagogisch fruchtbare von pädagogisch unfruchtbaren Inhalten zu unterscheiden.

Trotz aller Legitimationsnöte, geographiedidaktischer Vielfalt und inhaltlicher Unüberschaubarkeit des Geographieunterrichts scheint eine Einigung über die Grundintentionen geographischer Bildung und Erziehung in den Bereichen Natur bzw. Umwelterziehung, Wahrnehmung von Ländern und Völkern bzw. internationale Verständigung und Friedenserziehung, regionale und internationale Identität bzw. Heimaterziehung, sozialräumliche Disparitäten bzw. multikulturelle und politische Erziehung, internationale Arbeitsteilung bzw. Solidarität mit der Dritten Welt möglich. Aus diesem Grunde wurde vom Autor während des o. a. Symposiums in Salzburg angeregt, eine europäische Konferenz der Geographiedidaktiker durchzuführen, um den europäischen Ideenreichtum zu nutzen und die geographische Bildung der Bevölkerung Europas zu stärken.

\section{Endogene Philosophien des Geographie- unterrichtes in Entwicklungsländern}

Sowohl Vertreter aus Industrieländern als auch Vertreter aus Entwicklungsländern beschäftigen sich mehr und mehr mit spezifischen didaktischen Problemen des Geographieunterrichts in Entwicklungsländern. Christine Speak analysierte die Situation in einem Paper während des Kongresses 1988 in Sydney. Sie belegte und beklagte den Tatbestand, daß reiche Länder sich $z$. B. mehr und mehr mit interaktivem Video und "Computer-assisted learning» beschäftigen, währenddem Geographielehrern in Entwicklungsländern häufig die einfachsten Hilfsmittel fehlten; daß in Entwicklungsländern das Auswendiglernen von Fakten vorherrsche und in Industrieländern hingegen die Entwicklung formaler Fähigkeiten zur Partizipation an gesellschaftlichen Entwicklungen größere Bedeutung erhalte.

Trotz dieses Tatbestandes wird aber die Bemühung einiger Entwicklungsländer, sich auf die eigene Geschichte, Philosophie und Kultur zu besinnen, deutlich. Während z. B. in kolonialer Zeit und zum Teil auch noch heute die Curricula der Entwicklungsländer auf ihre «Mutterländer» ausgerichtet waren und 
sind, bemühen sich doch nun viele Entwicklungsländer, ihr eigenes Schicksal, Volk und Land in den Mittelpunkt des Schulunterrichts zu stellen. Was das konkret bedeutet, kann z. B. ein Zitat aus einem indischen Schülerbuch veranschaulichen. Hier heißt es: "Indien ist mein Land. Alle Inder sind meine Brüder und Schwestern. Ich liebe mein Land und ich bin stolz auf seine reiche und vielfältige Kultur. Ich werde alles tun, um ihrer würdig zu sein. Ich werde meinen Eltern, Lehrern und allen älteren Menschen Respekt erweisen und jedem mit Höflichkeit begegnen. Meinem Land und Volk bin ich ergeben. In seinem Wohlergehen allein liegt mein Glück.» (Maharashtra 1984)

Noch interessanter ist der Versuch einer philosophischen Untermauerung der Selbstbesinnung in Entwicklungsländern, wie es z. B. in einer Abhandlung von Josef Ouma, Uganda (in: HAUBRICH, H. (Hg.), Freiburg 1988, S. 10ff.) deutlich wird.

Ouma geht davon aus, daß eingeborene Afrikaner schon immer ihre eigene Umwelt gut verstanden haben. Philosophisch betrachtet analysierten die präkolonialen Afrikaner ihre Umwelt immer ganzheitlich. Sie unterteilten ihre Umwelt nicht in Teilgebiete. Hatten sie nur einen Teil im Blick, so suchten sie dadurch immer zum Ganzen zu gelangen. Ihr Interesse war immer integrativ bzw. ganzheitlich. Die Eingeborenen verstanden ihre Umwelt als ein zusammengehöriges Ganzes, also völlig anders als in der gegenwärtigen mikro-spezialisierten Betrachtung der Geographie. Dieser aktuelle geographische Ansatz wird als hinderlich für das Verständnis des Ganzen angesehen. Er trübe das wissenschaftliche Auge. Der wesentliche Unterschied in beiden Vorgehensweisen liegt darin, daß die traditionelle afrikanische Umweltbetrachtung vom «Ganzen zu den Teilen» geht und die moderne wissenschaftliche «von den Teilen zum Ganzen».

Der Afrikaner habe eine sehr tiefe Beziehung zu seiner Umwelt, d. h. seinem Geburtsort und seiner Heimat, wo er seine Jugend verbracht habe. Diese Umwelt wird von ihm individualisiert und personifiziert. Sie erhält von ihm einen Rufnamen, ja sogar mehrere Kosenamen. Diese Kosenamen spiegeln Lob und Preis, tiefe Zuneigung und Dank. Auch da, wo diese Umwelt eine unwirtliche Gegend darstellt, entdeckt der Afrikaner noch Gutes und Schätzenswertes. Diese tiefe Zuneigung zum eigenen Lebensraum erbringe das gleiche hohe Interesse, wenn dieser Raum zum Gegenstand von Unterricht gemacht werde. Die hohe Motivation führe nicht nur zum intellektuellen Interesse, sondern auch zum aktiven Engagement im eigenen Lebensraum. Ouma vergleicht die Mensch-Raum-Beziehungen eines Afrikaners mit einer Mutter-Kind-Beziehung, fügt aber sofort hinzu, daß diese Zuneigung grundsätzlich unbeschreibbar bleibe, und deshalb aber fundamental für das Umweltverständnis und die Lebensphiloso- phie eines eingeborenen Afrikaners. Obwohl ohne Schriftüberlieferung aufgewachsen, sei dieser in der Lage, seine historische und geographische Existenz zu erfassen und zu schätzen. Ein 18- bis 20jähriges Mädchen sei zu einer meisterhaften Kenntnis und Beherrschung ihrer Heimat befähigt, dabei habe sie formale Qualifikationen erlangt, die dazu führen, daß sie nach ihrer Heirat und Abwanderung in eine fremde Gegend diese schnell erschließen und zu ihrer zweiten Heimat werden lassen kann. Das Prinzip der personalen und emotionalen Beziehung zur Umwelt und des lebenslangen Lernens wird von Ouma als ein kardinales Prinzip geographischer Erziehung angesehen, das zum «Meister» seiner Umwelt befähige.

Der eingeborene Afrikaner betrachte aber nicht nur seinen eigenen Lebensraum als eine Einheit, sondern sehe ihn auch eingebettet in die Einheit des Universums. Er führt die unbelebte, belebte und geistige Welt auf einen gemeinsamen Ursprung mit einem gemeinsamen Ziel der Existenz zurück. Die Kraft, die das Universum zusammenhält, wird als «Spirit» bezeichnet. Kausales Denken war daher ein wesentlicher Bestandteil der afrikanischen Kultur. Ursache und Wirkung standen aber nicht isoliert da, sondern waren stets in ein universelles Ganzes eingebettet. Physische und metaphysische Erklärungen der Welt ergänzten einander. Auf diese Weise erhielt die «Welt-Anschauung» des Afrikaners eine große Tiefe.

Es ist hier nicht der Raum verfügbar, die gesamte Philosophie Oumas wiederzugeben. Die skizzenhaften Ausführungen mögen jedoch genügen, um zu zeigen, wie Afrikaner versuchen, «Afrika wiederzuentdecken», d. h. endogene afrikanische Weltanschauungen auch in die geographische Wissenschaft und in den geographischen Unterricht zu integrieren, um die Jugend zu einer vertieften Kenntnis ihres eigenen Handlungsraumes, zu einer starken Identität mit ihrer Heimat und zu einem «meisterhaften» Handeln in ihrer Umwelt zu befähigen.

\section{Struktur und Projekte der Kommission «Geographical Education» der «Internationalen Geographischen Union»}

Die internationale Entwicklung in der Geographiedidaktik zu beobachten und zu fördern, ist die zentrale Aufgabe der Kommission «Geographical Education» der Internationalen Geographischen Union. Während des Kongresses in Sydney und Brisbane 1988 waren z. B. über 150 von über 400 korrespondierenden Mitgliedern der Kommission vertreten, von denen 70 ein Paper, das eine geographiedidaktische Fragestellung zum Gegenstand hatte, zur Diskussion stellten. Alle Abhandlungen 
sind in den «Proceedings ...» hrsg. von Rod Gerber, College of Advanced Education, Brisbane 1988 veröffentlicht.

Während der Generalversammlung der Internationalen Geographischen Union wurde auf der Grundlage des Rechenschaftsberichts über die letzte Periode und der zukünftigen Arbeitspläne für die nächsten vier Jahre über den Fortbestand der Kommission positiv entschieden und der Autor dieses Aufsatzes zum Vorsitzenden gewählt. Zur Zeit sind die Verhandlungen über die $10+1$ möglichen full members der Kommission mit dem Präsidenten und Generalsekretär der IGU im Gange. Der neue Vorsitzende hat nach Rücksprache mit zahlreichen Mitgliedern die folgenden full members vorgeschlagen: Savita Sinha/Indien (Sekretärin), Suresh Garsole/ Indien, Rod Gerber/Australien, Patricia GreenMilberg/Canada, Erisa Kyangulanyi/Uganda, Vladimir Maksakowsky/UdSSR, Michael Morrissey/Jamaica, Michael Naish/Großbritannien, Julie Okpala/Nigeria, Joe Stoltman/USA, Lan-Sheng Zhang/China. Die Versammlung dieser Kommission von full members entscheidet z. B. über die Anerkennung von Forschungsprojekten und über zukünftige Symposia, und jedem einzelnen Mitglied ist die federführende Verantwortung für Teilbereiche der Geographiedidaktik übertragen. Solche Bereiche sind z.B.: Internationale Verständigung, Frauen im Geographieunterricht, Schulbuchforschung, geographiedidaktische Datenbank, Medienforschung, Lehrerbildung, Geographieunterricht in Entwicklungsländern, Curriculumforschung, Schulkartographie, Weltgeographie.

Einzelne full und corresponding members bzw. Teams bearbeiten zur Zeit folgende Projekte:

Philip Stimpson: Assessment and Curriculum, Kit Salter: Strategies promoting Geographical Education, Michael Naish: Centralization of Curricula, John Lidstone: Hazard Education, Julie Okpala: Map Skills, David Shape: Translating Educational Software, Michael Williams: Distance Learning, Ashley Kent: Recording Classroom Activities, Günter Niemz: International Testing, Lucile Marbeau: Initial and Inservice Training, H. Schrettenbrunner: Empirical Research, Hartwig Haubrich: Charter on Geographic Literacy.

Aus den zahlreichen Projekten sei nur ein konkretisierender Hinweis auf das letzte Projekt «Charta on Geographic Literacy» erlaubt. Viele Entwicklungsländer haben keine entsprechenden Ressourcen, um ihre Lehrpläne zu modernisieren, aber auch viele Industrieländer sind oft in Bedrängnis und erwarten Hilfe bei drohenden Einschränkungen des Geographieunterrichts durch internationale Institutionen. Nicht zuletzt deshalb, aber auch um nationale Erfahrungen international fruchtbar zu machen, wird ein Projekt begonnen, das auf der Grundlage der Analyse der geographiedidaktischen
Situation in der Welt und mit Hilfe international anerkannter Dokumente wie z. B. der Erklärung der Menschenrechte, der Charta der Rechte des Kindes, der Umweltcharta, der Schlußakte von Helsinki usw. eine Charta, d. h. eine internationale Erklärung über die Bedeutung der geographischen Bildung vereinbart. Aus dem Menschenrecht auf Bildung soll das Recht auf geographische Bildung für Jugendliche und Erwachsene abgeleitet, aber auch auf die Verpflichtung für ein umweltgerechtes und global-solidarisches Verhalten hingewiesen werden. Zur Realisierung dieses Projektes ist eine sehr breite internationale Beteiligung der Geographiedidaktiker notwendig, aber auch die Unterstützung von anderen internationalen Institutionen wie z. B. Europarat und UNESCO gefragt.

Die folgenden Projekte wurden im Jahre 1988 abgeschlossen:

1. Übersetzung der New UNESCO Source Book in the Teaching of Geography ins Russische

2. Unterrichtsmaterialien und Lehrerhinweise über «Land, Water, and Mineral Ressources» - ICSU Press London 1988

3. German Didactics of Geography in the 70ties and 80ties. A Review of Trends and Endavours - edited by Joseph Birkenhauer and Bill Marsden, München 1988

4. International Trends in Geographical Education edited by Hartwig Haubrich, Freiburg 1988

5 . How I see my country - edited by Hartwig Haubrich, Freiburg 1988

6. Video-Project Global Geography - Kalamazoo 1988

Um die Arbeit der Kommission Geographical Education zu verbreitern, wird zur Zeit eine Gruppe sogenannter «Regional Correspondents» gebildet. Diese informieren die Kommission über die aktuellen Ereignisse des eigenen Landes oder der Nachbarländer. Sie versuchen Kontakte zu entwickeln bzw. Aktivitäten von Geographiedidaktikern in ihrer Region zu unterstützen. Sie suchen die Zusammenarbeit mit allen Nachbarländern, die daran Interesse zeigen. Auf diese Art und Weise arbeiten die regionalen Korrespondenten mit ihren Kollegen, ihren nationalen, aber auch internationalen Geographiegesellschaften zusammen, um den Geographieunterricht, d. h. die geographische Bildung für Jugendliche und Erwachsene zu fördern und eine Brücke zwischen ihrer Region und der Kommission zu bilden. Sie versuchen, wichtige Informationen aus den zweimal jährlich erscheinenden Newsletters in ihrer nationalen geographischen Zeitschrift zu veröffentlichen. Alle zwei Jahre während der regional conferences und der main congresses (1990 in Beijing, 1992 in Washington) wird die Arbeit der regional correspondents von der Kommission ausgewertet und neu durchdacht. 


\section{Zusammenfassung}

Welttrends in der Geographiedidaktik werden nicht deutlich erkennbar. Die Dezentralisierung in der Sowjetunion hat dagegen eindeutig westliche Vorbilder. Die Zentralisierung in Großbritannien folgt einem konservativen Trend, der vielen westlichen Ländern gemeinsam ist und orientiert sich an französischen Modellen. Die Unübersichtlichkeit in «östlichen» und «westlichen» europäischen Ländern entspricht der vorhandenen politischen Zersplitterung und Kleinkammerung Europas. Während fortschrittliche Industrienationen sich um die Entwicklung von interaktivem Video und Computer assisted Learning bemühen, fehlen den meisten Entwicklungsländern die einfachsten Lehr- und Lernmittel. Eine Zweiklassendidaktik, die mittlerweile bereits Realität geworden ist, wurde von Christine Speak in Sydney 1988 beschrieben und angeklagt. Die Besinnung auf die eigene Kultur und die Entwicklung seiner eigenständigen geographiedidaktischen Philosophie zeigen sich dem Autor zögernd in Uganda und in Indien. Zur Fortentwicklung der Geographiedidaktik hat gerade die Kommission «Geographical Education» der Internationalen Geographischen Union ihren Arbeitsplan für die Periode 1988-92 vorgelegt.

\section{Literatur}

BIRKENHAUER, J. und MARSDEN, B. (Hrsg), (1988): German Didactics of Geography in the 70ties and 80ties, München.

GERBER, R. (Hrsg), (1988): Proceedings of the Symposium of the Commission Geographical Education, 1988, Brisbane.

GRAVES, A. (ed.), (1982): New Unesco Textbook for Geography Teaching, Longman, Paris.

HAUBRICH, H. (Hrsg), (1982): Geographische Erziehung im internationalen Blickfeld, Braunschweig.

HAUBRICH, H. (Hrsg), (1987): International Trends in Geographical Education, Freiburg.

HAUBRICH, H. (Hrsg), (1988): How I see my country, Freiburg.

HAUBRICH, H. u. a. (1988): Didaktik der Geographie - konkret, Oldenburg/München.

MAHARASHTRA STATE BUREAU of Textbook, Production and Curriculum Research (Hrsg.) (1984): Our neighbours Our World. Maharshtra.

SPERLING, W. (1977): Geographieunterricht und politische Bildung und Erziehung in der DDR. In: Frankfurter Beiträge zur Did. der Geographie, Bd. 1, S. 208-228, Frankfurt/M.

WOHLSCHLÄGL, H. (Hrsg), (1989): Bericht über das Mitteleuropäische Geographiedidaktik Symposium während des Deutschen Schulgeographentages in Salzburg 1988 mit Beiträgen aus der Bundesrepublik Deutschland, der CSSR, der DDR, der Niederlande, Österreich, Polen, Schweiz und Ungarn, Wien. 